\title{
Symmetry Enriched U(1) Topological Orders for Dipole-Octupole Doublets on a Pyrochlore Lattice
}

\author{
Yao-Dong $\mathrm{Li}^{1}$ and Gang Chen ${ }^{1,2 *}$ \\ ${ }^{1}$ State Key Laboratory of Surface Physics, Center for Field Theory and Particle Physics, \\ Department of Physics, Fudan University, Shanghai 200433, People's Republic of China and \\ ${ }^{2}$ Collaborative Innovation Center of Advanced Microstructures, Nanjing, 210093, People's Republic of China
}

(Dated: August 28, 2018)

\begin{abstract}
Symmetry plays a fundamental role in our understanding of both conventional symmetry breaking phases and the more exotic quantum and topological phases of matter. We explore the experimental signatures of symmetry enriched U(1) quantum spin liquids (QSLs) on the pyrochlore lattice. We point out that the Ce local moment of the newly discovered pyrochlore QSL candidate $\mathrm{Ce}_{2} \mathrm{Sn}_{2} \mathrm{O}_{7}$, is a dipole-octupole doublet. The generic model for these unusual doublets supports two distinct symmetry enriched U(1) QSL ground states in the corresponding quantum spin ice regimes. These two U(1) QSLs are dubbed dipolar U(1) QSL and octupolar U(1) QSL. While the dipolar U(1) QSL has been discussed in many contexts, the octupolar U(1) QSL is rather unique. Based on the symmetry properties of the dipole-octupole doublets, we predict the peculiar physical properties of the octupolar U(1) QSL, elucidating the unique spectroscopic properties in the external magnetic fields. We further predict the Anderson-Higgs transition from the octupolar U(1) QSL driven by the external magnetic fields. We identify the experimental relevance with the candidate material $\mathrm{Ce}_{2} \mathrm{Sn}_{2} \mathrm{O}_{7}$ and other dipole-octupole doublet systems.
\end{abstract}

Introduction.-The interplay between symmetry and topology is the frontier subject in modern condensed matter physics [1-3]. At the single particle level, the non-trivial realization of time reversal symmetry in electron band structure has led to the discovery of topological insulators $[4,5]$. For the intrinsic topological order such as $\mathrm{Z}_{2}$ toric code and chiral Abelian topological order, a given symmetry of the system could enrich the topological order into distinct phases that cannot be smoothly connected without crossing a phase transition [6-9]. Despite the active theoretical efforts, the experimentally relevant symmetry enriched topological order is extremely rare. In this work, we explore one physical realization of symmetry enriched U(1) topological order for the dipole-octupole (DO) doublets on the pyrochlore lattice and predict the experimental consequences of distinct symmetry enrichment. The DO doublet is a special Kramers' doublet in the $\mathrm{D}_{3 \mathrm{~d}}$ crystal field environment [10-12]. Both states of the DO doublet transform as the one-dimensional irreducible representations $\left(\Gamma_{5}^{+}\right.$or $\left.\Gamma_{6}^{+}\right)$of the $\mathrm{D}_{3 \mathrm{~d}}$ point group [10]. It was realized that the DO doublets on the pyrochlore lattice could support two distinct $\mathrm{U}(1)$ quantum spin liquid (QSL) ground states [10]. These distinct U(1) QSLs are the symmetry enriched U(1) topological orders [13] and are enriched by the lattice symmetries of the pyrochlore systems.

Recently $\mathrm{Ce}_{2} \mathrm{Sn}_{2} \mathrm{O}_{7}$ was proposed as the first Ce-based QSL candidate in the pyrochlore family [14], in which no magnetic order was observed down to $0.02 \mathrm{~K}$. Although it was not noticed previously, the $\mathrm{Ce}^{3+}$ local moment in $\mathrm{Ce}_{2} \mathrm{Sn}_{2} \mathrm{O}_{7}$ is actually a DO doublet. The strong atomic spin-orbit coupling (SOC) of the $4 f^{1}$ electron in the $\mathrm{Ce}^{3+}$ ion entangles the electron spin $(S=1 / 2)$ with the orbital angular momentum $(L=3)$ into a $J=5 / 2$ total mo-

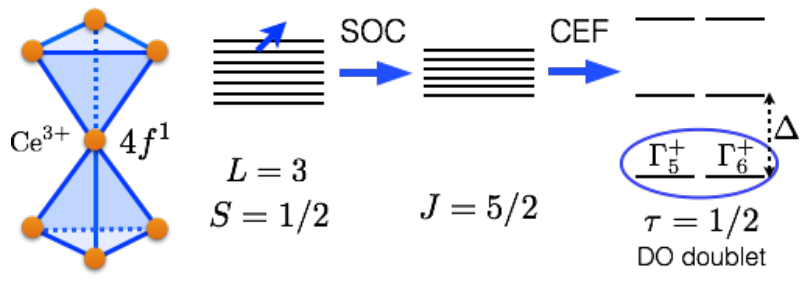

FIG. 1. The electron configuration and the $\mathrm{D}_{3 \mathrm{~d}}$ crystal electric field $(\mathrm{CEF})$ splitting of the $\mathrm{Ce}^{3+}$ ion in $\mathrm{Ce}_{2} \mathrm{Sn}_{2} \mathrm{O}_{7}$. The CEF ground state wavefunctions are combinations of $J^{z}=$ $\pm 3 / 2$ states [14], thus the CEF ground state is a DO doublet. $\Delta$ is the CEF gap and was fitted to be $\Delta=50 \pm 5 \mathrm{meV}$ [14].

ment. The six-fold degeneracy of the $J=5 / 2$ total moment is further splitted into three Kramers' doublets by the $\mathrm{D}_{3 \mathrm{~d}}$ crystal field (see Fig. 1). Since the ground state doublet wavefunctions are combinations of $J^{z}= \pm 3 / 2$ states [14], this doublet is precisely the DO doublet that we defined [10]. Because the crystal field gap is much larger than the interaction energy scale of the local moments and the temperature scale in the experiments, the low temperature magnetic property of $\mathrm{Ce}_{2} \mathrm{Sn}_{2} \mathrm{O}_{7}$ is fully governed by the ground state doublets.

Motivated by the experiments on $\mathrm{Ce}_{2} \mathrm{Sn}_{2} \mathrm{O}_{7}$ and more generally by the experimental consequences of the distinct symmetry enriched U(1) QSLs for the DO doublets, in this Letter, we explore the peculiar properties of the DO doublets in external magnetic fields. In the octupolar U(1) QSL of the octupolar quantum spin ice regime for the DO doublets, we find that the external magnetic field directly couples to the spinons and modifies the spinon dispersions. This effect allows us to control the spinon excitations with the magnetic fields. The lower excitation edge of the spinon continuum in the dynamic spin struc- 
(a) Phase diagram for $h \|[111]$

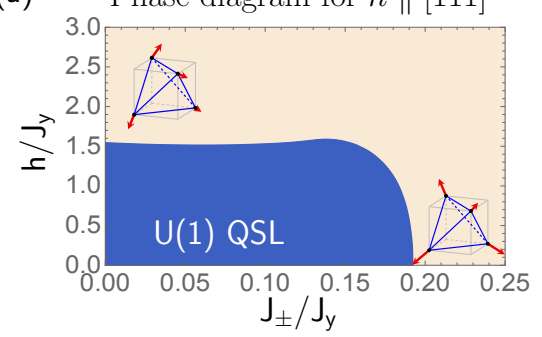

(b) Phase diagram for $h \|[001]$

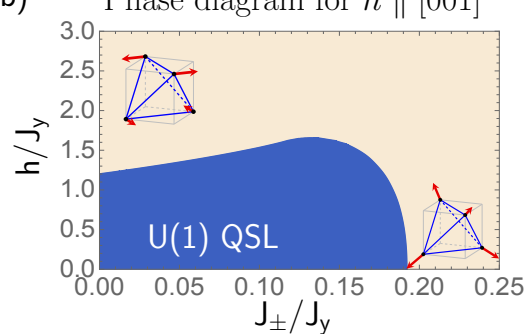

(c) Phase diagram for $h \|[110]$

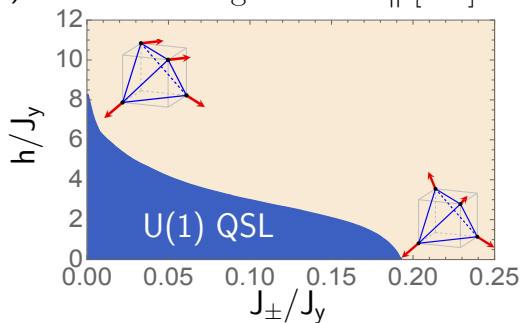

FIG. 2. Phase diagrams for magnetic fields along (a) [111], (b) [001], and (c) [110] directions. Outside the QSL phases are the induced magnetic ordered phase via the spinon condensation. For $h=0$, the spinons are condensed at $\mathbf{k}_{c}=(0,0,0)$, and we choose the local moments to order in the local $\hat{z}$ direction. In (a), large magnetic field near the vertical axis drives the spinon condensation at $\mathbf{k}_{c}=\pi(1,1,1)$, and the resulting order is depicted in the figure. This order smoothly connects to the order on the horizontal axis. The cases in (b) and (c) are similar, except that in (b) the field on the vertical axis drives the condensation at $\mathbf{k}_{c}=2 \pi(0,0,1)$, while in (c) $\mathbf{k}_{c}=\pi(1,1,0)$ near the vertical axis. We set the diamond lattice constant to unity.

ture factors can thus be modified by the magnetic fields, which gives a sharp prediction for the inelastic neutron scattering experiments. When the magnetic field exceeds the critical value and closes the spinon gap, the spinons are condensed, driving the system through an AndersonHiggs' transition and inducing the long-range magnetic orders.

Generic model for DO doublets on the pyrochlore lattice.-Because of the peculiar symmetry properties of the DO doublets, the most generic model that describes the nearest-neighbor interaction between them is given as $H_{\mathrm{DO}}=\sum_{\langle i j\rangle}\left[J_{x} \tau_{i}^{x} \tau_{j}^{x}+J_{y} \tau_{i}^{y} \tau_{j}^{y}+J_{z} \tau_{i}^{z} \tau_{j}^{z}+J_{x z}\left(\tau_{i}^{x} \tau_{j}^{z}+\right.\right.$ $\left.\tau_{i}^{z} \tau_{j}^{x}\right)$ ] [10]. Here the interaction is uniform on every bond despite the fact that the DO doublet involves a significant contribution from the orbital part due to the strong SOC [15-20], and the DO doublet is modeled by an effective pseudospin- $1 / 2$ moment $\boldsymbol{\tau}$. Both $\tau^{x}$ and $\tau^{z}$ transform as the dipole moments under the space group symmetry, while the $\tau^{y}$ component behaves as an octupole moment [10]. It is this important difference that leads to some of the unique properties of its U(1) QSL ground states.

Due to the spatial uniformity of the generic model, we can transform the model $H_{\mathrm{DO}}$ into the $\mathrm{XYZ}$ model with

$$
H_{\mathrm{XYZ}}=\sum_{\langle i j\rangle} \tilde{J}_{x} \tilde{\tau}_{i}^{x} \tilde{\tau}_{j}^{x}+\tilde{J}_{y} \tilde{\tau}_{i}^{y} \tilde{\tau}_{j}^{y}+\tilde{J}_{z} \tilde{\tau}_{i}^{z} \tilde{\tau}_{j}^{z}
$$

where $\tilde{\tau}^{x}$ and $\tilde{\tau}^{z}\left(\tilde{J}_{x}\right.$ and $\left.\tilde{J}_{z}\right)$ are related to $\tau^{x}$ and $\tau^{z}$ $\left(J_{x}\right.$ and $\left.J_{z}\right)$ by a rotation around the $y$ direction in the pseudospin space, and $\tilde{\tau}^{y} \equiv \tau^{y}, \tilde{J}_{y} \equiv J_{y}$. When one of the couplings, $\tilde{J}_{\mu}$, is dominant and antiferromagnetic, the corresponding pseudospin component, $\tilde{\tau}^{\mu}$, is regarded as the Ising component of the model, and the ground state is a U(1) QSL in the corresponding quantum spin ice regime. The dipolar U(1) QSL is realized when the Ising component is the dipole moment $\tilde{\tau}^{x}$ or $\tilde{\tau}^{z}$, while the octupolar U(1) QSL is realized when the Ising component is the octupole moment $\tilde{\tau}^{y}$. In the compact $\mathrm{U}(1)$ quantum electrodynamics description of the low energy properties of the U(1) QSL [21, 22], the Ising component is identified as the emergent electric field [21]. Therefore, the emergent electric field transforms very differently under the lattice symmetry in dipolar and octupolar U(1) QSLs, making these two $\mathrm{U}(1)$ QSLs symmetry enriched $\mathrm{U}(1)$ topological order on the pyrochlore lattice [10].

Octupolar U(1) QSL and field-driven Anderson-Higgs' transitions. - Since the dipolar U(1) QSL has been discussed many times in literature [10,23-31], we here focus on the octupolar U(1) QSL of the octupolar quantum spin ice regime where $\tilde{J}_{y}$ is dominant and antiferromagnetic. The octupolar U(1) QSL is a new phase that is unique to the DO doublet and cannot be found in any other doublets on the pyrochlore lattice.

We consider the coupling of the DO doublet to the external magnetic field. Remarkably, because $\tilde{\tau}^{y}$ is an octupole moment, it does not couple to the magnetic field even though it is time reversally odd. Only the dipolar component, $\tau^{z}$, couples linearly to the external magnetic field. The resulting model is

$$
H=\sum_{\langle i j\rangle} \sum_{\mu=x, y, z} \tilde{J}_{\mu} \tilde{\tau}_{i}^{\mu} \tilde{\tau}_{j}^{\mu}-\sum_{i} h\left(\hat{n} \cdot \hat{z}_{i}\right) \tau_{i}^{z}
$$

where $\hat{n}$ is the direction of the magnetic field and $\hat{z}_{i}$ is the $z$ direction of the local coordinate basis at the lattice site $i$ [32]. This generic model describes all magnetic properties of the DO doublets on the pyrochlore lattice.

As the generic model contains four parameters, it necessarily brings some unnecessary complication into the problem. To capture the essential physics, we here consider a simplified version of the generic model in Eq. (2). The simplified model is

$$
\begin{aligned}
H_{\mathrm{sim}} & =\sum_{\langle i j\rangle} J_{y} \tau_{i}^{y} \tau_{j}^{y}-J_{ \pm}\left(\tau_{i}^{+} \tau_{j}^{-}+h . c .\right) \\
& -\sum_{i} h\left(\hat{n} \cdot \hat{z}_{i}\right) \tau_{i}^{z}
\end{aligned}
$$

where we define $\tau_{i}^{ \pm}=\tau_{i}^{z} \pm i \tau_{i}^{x}$ and $\hat{n}$ is the direction of the external magnetic field. In the Ising limit with 
(a)

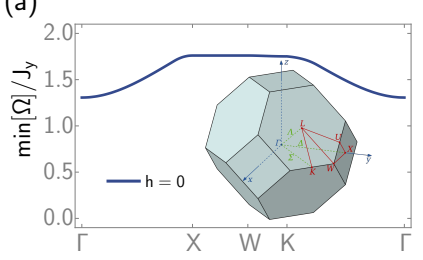

(c)

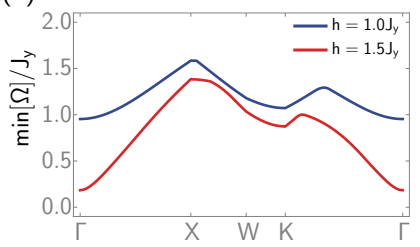

(b)

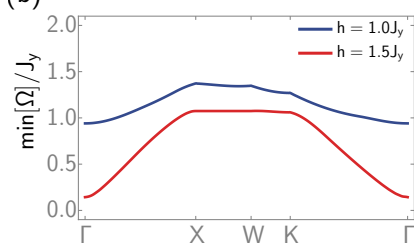

(d)

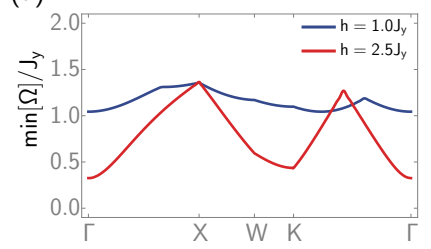

FIG. 3. Lower excitation edges of the spinon continuum in the dynamic spin structure factor under (a) zero magnetic field, and field along (b) [111], (c) [001], and (d) [110] directions.. In the figure, we set $J_{ \pm}=0.1 J_{y}$. The inset of (a) is the Brillouin zone [33].

$J_{ \pm}=0$ and $h=0$, the antiferromagnetic $J_{y}$ favors the $\tau^{y}$ components to be in the ice manifold and requires a "two-plus two-minus" ice constraint for the $\tau^{y}$ configuration on each tetrahedron. This octupolar ice manifold is extensively degenerate. With a small and finite $J_{ \pm}$ or $h$, the system can then tunnel quantum mechanically within the octupolar ice manifold and form an octupolar U(1) QSL. In this perturbative limit, the degenerate perturbation theory yields an effective ring exchange model with [32]

$$
H_{\text {ring }}=J_{\text {ring }} \sum_{\bigcirc}\left[\tau_{i}^{+} \tau_{j}^{-} \tau_{k}^{+} \tau_{l}^{-} \tau_{m}^{+} \tau_{n}^{-}+\text {h.c. }\right],
$$

where " $i, j, k, l, m, n$ " are six sites on the perimeter of the elementary hexagon of the pyrochlore lattice, and the ring exchange $J_{\text {ring }}<0$ for $J_{ \pm}>0$ and for either sign of $h$. $H_{\text {ring }}$ does not involve defect tetrahedra that violate the ice constraint and thus only describes the quantum fluctuation and dynamics within the ice manifold. It is well-known that the low energy properties of $H_{\text {ring }}$ is described by the compact $\mathrm{U}(1)$ quantum electrodynamics [21] of the U(1) QSL with gapless gauge photon, and the spin-flip operator $\tau_{i}^{ \pm}$is identified as the gauge string within the ice manifold. We expect the simplified model $H_{\text {sim }}$ captures the generic properties of the octupolar U(1) QSL.

To obtain the phase diagram of $H_{\text {sim }}$, we start from the octupolar U(1) QSL phase and study its instability. For this purpose, we include the spinon excitations (that are out of the ice manifold) into the formulation. The perturbative analysis and $H_{\text {ring }}$, that focus on the ice manifold, does not capture the spinons. We here implement a parton-gauge construction for the octupolar $\mathrm{U}(1)$ QSL and formulate $H_{\text {sim }}$ into a lattice gauge theory with the spinons. Like many other parton construction, we

replace the physical Hilbert space with a larger one and supplement it with a constraint. We follow Refs. 23 and 24 and express the pseudospin operators as

$$
\tau_{i}^{+}=\Phi_{\mathbf{r}}^{\dagger} \Phi_{\mathbf{r}^{\prime}} s_{\mathbf{r r}^{\prime}}^{+}, \quad \tau_{i}^{y}=s_{\mathbf{r r}^{\prime}}^{y},
$$

where $\mathbf{r r}^{\prime}$ is the link that connects two neighboring tetrahedral centers at $\mathbf{r}$ and $\mathbf{r}^{\prime}$, and the pyrochlore site $i$ is shared by the two tetrahedra. The centers of the tetrahedra form a diamond lattice, and $\mathbf{r}\left(\mathbf{r}^{\prime}\right)$ belongs to the I (II) diamond sublattice. Here $\mathbf{s}_{\mathbf{r} \mathbf{r}^{\prime}}$ is a spin- $1 / 2$ variable that corresponds to the emergent gauge field, and $\Phi_{\mathbf{r}}^{\dagger}\left(\Phi_{\mathbf{r}}\right)$ creates (annihilates) one spinon at the diamond site $\mathbf{r}$. The spinons carry the emergent electric charge, and $\Phi_{\mathbf{r}}^{\dagger}$ and $\Phi_{\mathbf{r}}$ are raising and lowering operators of the emergent electric charge. Since we enlarged the physical Hilbert space, the constraint $Q_{\mathbf{r}}=\eta_{\mathbf{r}} \sum_{\mu} \tau_{\mathbf{r}, \mathbf{r}+\eta_{\mathbf{r}} \mathbf{e}_{\mu}}^{y}$ is imposed, where $\eta_{\mathbf{r}}=1(-1)$ for the I (II) sublattice and the $\mathbf{e}_{\mu}$ 's are the first neighbor vectors of the diamond lattice. Here $Q_{\mathbf{r}}$ measures the electric charge at $\mathbf{r}$ and satisfies

$$
\left[\Phi_{\mathbf{r}}, Q_{\mathbf{r}}\right]=\Phi_{\mathbf{r}}, \quad\left[\Phi_{\mathbf{r}}^{\dagger}, Q_{\mathbf{r}}\right]=-\Phi_{\mathbf{r}}^{\dagger} .
$$

The U(1) QSL of quantum spin ice is an example of the string-net condensed phases [34]. In the U(1) QSL, $\tau_{i}^{ \pm}$ creates the shortest open (gauge) string whose ends are spinon particles. In the spin ice context, $\tau_{i}^{ \pm}$creates two defect tetrahedra that violate the "two-plus two-minus" ice constraint. The parton-gauge construction captures this essential property, and the model becomes

$$
\begin{aligned}
H_{\text {sim }} & =\sum_{\mathbf{r}} \frac{J_{y} Q_{\mathbf{r}}^{2}}{2}-\sum_{\mathbf{r}} \sum_{\mu \neq \nu} J_{ \pm} \Phi_{\mathbf{r}+\eta_{\mathbf{r}} \mathbf{e}_{\mu}}^{\dagger} \Phi_{\mathbf{r}+\eta_{\mathbf{r}} \mathbf{e}_{\nu}} s_{\mathbf{r}, \mathbf{r}+\eta_{\mathbf{r}} \mathbf{e}_{\mu}}^{-\eta_{\mathbf{r}}} \\
& \times s_{\mathbf{r}, \mathbf{r}+\eta_{\mathbf{r}} \mathbf{e}_{\nu}}^{+\eta_{\mathbf{r}}}-\sum_{\left\langle\mathbf{r} \mathbf{r}^{\prime}\right\rangle} \frac{h}{2}\left(\hat{n} \cdot \hat{z}_{i}\right)\left(\Phi_{\mathbf{r}}^{\dagger} \Phi_{\mathbf{r}^{\prime}} s_{\mathbf{r \mathbf { r } ^ { \prime }}}^{+}+\text {h.c. }\right) .
\end{aligned}
$$

With the constraint, Eq. (7) is an exact reformulation of the simplified model in Eq. (3). It describes the bosonic spinons hopping on the diamond lattice. The spinons are minimally coupled with the emergent $\mathrm{U}(1)$ gauge field. Remarkably, the external magnetic field directly couples to the spinons and does not couple to the emergent electric field. This is sharply distinct from the dipolar $\mathrm{U}(1)$ QSL where the magnetic field would also directly couple with the emergent electric field.

Inside the U(1) QSL, the spinons are fully gapped. The external magnetic field allows the spinon to tunnel between the neighbor tetrahedra that are located along the field direction. As we increase the magnetic field $h$, the spinon gap gradually decreases. It is expected that, at a critical field strength, the spinon gap is closed and the spinons are condensed with $\left\langle\Phi_{\mathbf{r}}\right\rangle \neq 0$. Via the AndersonHiggs' mechanism, the $\mathrm{U}(1)$ gauge field becomes massive and gapped. Note this differs the Coulomb ferromagnet where the gauge field remains gapless and deconfined [23]. The resulting proximate state develops a long-range magnetic order. Therefore, this is an Anderson-Higgs' transition driven by the external magnetic fields. This is a 
generic property of the octupolar U(1) QSL and is not a specific property of the simplified model. To our knowledge, this is the first example that an external probe drives an Anderson-Higgs' transition in a physical system.

To solve the reformulated model in Eq. (7), we adopt the gauge mean-field approximation [10, 23-25]. In this approximation, we decouple the model into the spinon sector and the gauge sector. Since $H_{\text {ring }}$ favors a zero background gauge flux on each elementary hexagon of the diamond lattice, we solve for the mean-field ground state within this sector [32]. The magnetic dipolar order is obtained by evaluating

$$
\begin{aligned}
\left\langle\tau_{i}^{z}\right\rangle & =\frac{1}{2}\left[\left\langle\tau_{i}^{+}\right\rangle+\left\langle\tau_{i}^{-}\right\rangle\right] \\
& =\frac{1}{2}\left[\left\langle\Phi_{\mathbf{r}}^{\dagger} \Phi_{\mathbf{r}^{\prime}}\right\rangle\left\langle s_{\mathbf{r r}^{\prime}}^{+}\right\rangle+\text {h.c. }\right],
\end{aligned}
$$

where $\langle\cdots\rangle$ is taken with respect to the ground state. Because of the Zeeman coupling, $\left\langle\tau_{i}^{z}\right\rangle$ is non-zero even in the U(1) QSL phase where the spinons are not condensed. In the proximate ordered state, the spinon condensate gives an additional contribution that is the induced magnetic order. For all three directions of the external magnetic field, even though the spinons are condensed at finite momenta, the proximate magnetic order preserves the translation symmetry.

The full phase diagrams and the field-induced proximate magnetic orders are depicted in Fig. 2. The magnetic field is found to be least effective in destructing the U(1) QSL for the field along the [110] direction. This is because the local $\hat{z}$ direction of two sublattices are orthogonal to the [110] direction and the pseudospins on them do not couple to the external field. The phase transition is found to be continuous within the gauge mean-field theory and may turn weakly first order after the fluctuations are included. Nevertheless, as the spinon gap is very small near the phase transition, this means that the heat capacity and the magnetic entropy will be more pronounced at low temperatures in these regions.

Lower excitation edges of the dynamic spin structure factors.- A smoking gun confirmation of U(1) QSL is to directly measure the gapless $\mathrm{U}(1)$ gauge photon and/or the spinon continuum by inelastic neutron scattering (INS) measurement. For the DO doublet, the neutron spin couples to the local moment in the same way as the external magnetic field. Therefore, for the octupolar U(1) QSL, the INS directly probes the spinon excitation, and one would only observe the spinon continuum instead of the gapless U(1) gauge photon. The latter was proposed for the dipolar U(1) QSL. This is the sharp difference between the octupolar U(1) QSL and the dipolar U(1) QSL.

In the U(1) QSL, the spinon excitation has two branches due to the two sublattice structure of the diamond lattice. Specifically for the simplified model $H_{\text {sim }}$, the two spinon branches are degenerate in the absence of the external magnetic field because the spinons do not hop from one sublattice to another. As shown in Eq. (7), however, the magnetic field allows the spinons to tunnel between the sublattices and breaks the degeneracy of the two spinon bands. The splitted spinon bands are labeled by $\omega_{1}(\mathbf{k})$ and $\omega_{2}(\mathbf{k})[32]$.

The INS measures the dynamic spin structure factor $\left\langle\tau^{z} \tau^{z}\right\rangle_{\mathbf{q}, \Omega}$, where $\mathbf{q}$ and $\Omega$ are the neutron momentum and energy transfer, respectively. As $\tau^{z}$ is a spinon bilinear, one neutron spin flip creates one spinon-antispinon pair that shares the neutron energy and momentum transfer. From the conservation of the momentum and the energy, we have

$$
\begin{aligned}
\mathbf{q} & =\mathbf{k}_{1}+\mathbf{k}_{2}, \\
\Omega(\mathbf{q}) & =\omega_{i}\left(\mathbf{k}_{1}\right)+\omega_{j}\left(\mathbf{k}_{2}\right),
\end{aligned}
$$

where $i, j=1,2$ are the band indices, and $\mathbf{k}_{1}$ and $\mathbf{k}_{2}$ are the momenta of the two spinons.

The lower excitation edge of the dynamic spin structure factor encodes the minimum of the spinon excitation $\Omega(\mathbf{q})$ for each q. In Fig. 3, we plot the dispersion of the lower spinon excitation edge along the high symmetric momentum direction in the octupolar U(1) QSL for different external field orientations. The field modifies the spinon dispersion and then tunes the spinon excitation edge. As far as we are aware of, this is a rare example that one can control the spinon excitations in a QSL.

Discussion.-Many DO doublet pyrochlores are actually magnetically ordered [35-42], which makes the QSL candidate $\mathrm{Ce}_{2} \mathrm{Sn}_{2} \mathrm{O}_{7}$ rather unique. $\mathrm{Ce}_{2} \mathrm{Sn}_{2} \mathrm{O}_{7}$ has the Curie-Weiss temperature $\Theta_{\mathrm{CW}} \approx-0.25 \mathrm{~K}$. It was argued in Ref. 14 that an antiferromagnetic $\Theta_{\mathrm{CW}}$ cannot support a QSL in the spin ice regime. This conclusion is certainly true for the usual Kramers' doublet, but is not the case for the DO doublets. For the DO doublets, what $\Theta_{\mathrm{CW}}$ measures is $J_{z}$, not $\tilde{J}_{z}$ nor $\tilde{J}_{x}$ [32]. What determines the phase diagram of $H_{\mathrm{XYZ}}$ are $\tilde{J}_{\mu}$ 's, not the sign or value of the single parameter $J_{z}$. One cannot rule out the possibility of the dipolar $\mathrm{U}(1)$ QSL in $\mathrm{Ce}_{2} \mathrm{Sn}_{2} \mathrm{O}_{7}$. Moreover, the occurrence of octupolar U(1) QSL as a ground state of $H_{\mathrm{XYZ}}$ is actually insensitive to the sign of $J_{z}$. If the ground state of $\mathrm{Ce}_{2} \mathrm{Sn}_{2} \mathrm{O}_{7}$ does not belong to any other QSLs, the question then nails down to whether it is a dipolar U(1) QSL or an octupolar U(1) QSL.

In Tab. I we list the thermodynamic and spectroscopic properties of various U(1) QSLs. Clearly, thermodynamic measurements cannot differentiate them because the low-energy properties are all described by the compact U(1) quantum electrodynamics. The INS measurement, however, is a powerful technique to identify the dipolar U(1) QSL and the octupolar U(1) QSL for the DO doublets. As we wrote in Tab. I, the INS can observe both spinon continuum and gapless gauge photon for the dipolar U(1) QSL while only gapped spinon continuum can be detected for the octupolar U(1) QSL. We further 


\begin{tabular}{ccc}
\hline \hline Different U(1) QSLs & Heat capacity & Inelastic neutron scattering measurement \\
Octupolar U(1) QSL for DO doublets & $C_{v} \sim T^{3}$ & Gapped spinon continuum \\
Dipolar U(1) QSL for DO doublets & $C_{v} \sim T^{3}$ & Both gapless gauge photon and gapped spinon continuum \\
Dipolar U(1) QSL for non-Kramers' doublets [24] & $C_{v} \sim T^{3}$ & Gapless gauge photon \\
Dipolar U(1) QSL for usual Kramers' doublets [23] & $C_{v} \sim T^{3}$ & Both gapless gauge photon and gapped spinon continuum \\
\hline \hline
\end{tabular}

TABLE I. List of the physical properties of different U(1) QSLs on the pyrochlore lattice. "Usual Kramers doublet" refers to the Kramers doublet that is not a DO doublet. They transform as a two-dimensional irreducible representation under the $\mathrm{D}_{3 \mathrm{~d}}$ point group. Although the dipolar U(1) QSL for DO doublets behaves the same as the one for usual Kramers' doublets, their physical origins are rather different [32].

propose the field driven Anderson-Higgs' transition and the field-controlled dynamic spin structure factor as the unique signatures of the octupolar U(1) QSL. All these prediction can be useful to identify the nature of the QSL ground state in $\mathrm{Ce}_{2} \mathrm{Sn}_{2} \mathrm{O}_{7}$.

To summarize, we predict a field driven AndersonHiggs' transition of the octupolar U(1) QSL for the dipole-octupole doublets on the pyrochlore lattice. Inside the U(1) QSL, the lower excitation edges of the spinon continuum are manipulated by the external magnetic fields. This result provides a detectable experimental consequence in the INS measurements. We expect our work will surely stimulate the experimental studies of $\mathrm{Ce}_{2} \mathrm{Sn}_{2} \mathrm{O}_{7}$ and other pyrochlore systems with dipoleoctupole doublets.

Acknowledgements. - This work is supported by the Start-up funds of Fudan University (Shanghai, People's Republic of China) and the Thousand-Youth-Talent program of People's Republic of China.

* gangchen.physics@gmail.com

[1] Zheng-Cheng $\mathrm{Gu}$ and Xiao-Gang Wen, "Tensorentanglement-filtering renormalization approach and symmetry-protected topological order," Phys. Rev. B 80, 155131 (2009).

[2] Frank Pollmann, Erez Berg, Ari M. Turner, and Masaki Oshikawa, "Symmetry protection of topological phases in one-dimensional quantum spin systems," Phys. Rev. B 85, 075125 (2012).

[3] T. Senthil, "Symmetry-protected topological phases of quantum matter," Annual Review of Condensed Matter Physics 6, 299-324 (2015).

[4] M Zahid Hasan and Charles L Kane, "Colloquium: topological insulators," Reviews of Modern Physics 82, 3045 (2010).

[5] Xiao-Liang Qi and Shou-Cheng Zhang, "Topological insulators and superconductors," Reviews of Modern Physics 83, 1057 (2011).

[6] Xiao-Gang Wen, Quantum Field Theory of Many-body Systems: From the Origin of Sound to an Origin of Light and Electrons (Oxford Graduate Texts), reissue ed. (Oxford University Press, USA, 2007).

[7] Andrej Mesaros and Ying Ran, "Classification of sym- metry enriched topological phases with exactly solvable models," Phys. Rev. B 87, 155115 (2013).

[8] Andrew M. Essin and Michael Hermele, "Classifying fractionalization: Symmetry classification of gapped $Z_{2}$ spin liquids in two dimensions," Phys. Rev. B 87, 104406 (2013).

[9] Yuan-Ming Lu and Ashvin Vishwanath, "Classification and properties of symmetry-enriched topological phases: Chern-simons approach with applications to $Z_{2}$ spin liquids," Phys. Rev. B 93, 155121 (2016).

[10] Yi-Ping Huang, Gang Chen, and Michael Hermele, "Quantum Spin Ices and Topological Phases from Dipolar-Octupolar Doublets on the Pyrochlore Lattice," Phys. Rev. Lett. 112, 167203 (2014).

[11] Yao-Dong Li, Xiaoqun Wang, and Gang Chen, "Anisotropic spin model of strong spin-orbit-coupled triangular antiferromagnets," Phys. Rev. B 94, 035107 (2016).

[12] Yao-Dong Li, Xiaoqun Wang, and Gang Chen, "Hidden multipolar orders of dipole-octupole doublets on a triangular lattice," Phys. Rev. B 94, 201114 (2016).

[13] We here adopt the definition of the U(1) topological order in Ref. 21. The concept is more general than the fully gapped intrinsic topological orders that are described by topological quantum field theory.

[14] Romain Sibille, Elsa Lhotel, Vladimir Pomjakushin, Chris Baines, Tom Fennell, and Michel Kenzelmann, "Candidate quantum spin liquid in the $\mathrm{Ce}^{3+}$ pyrochlore stannate $\mathrm{Ce}_{2} \mathrm{Sn}_{2} \mathrm{O}_{7}$, , Phys. Rev. Lett. 115, 097202 (2015).

[15] Gang Chen and Leon Balents, "Spin-orbit effects in $\mathrm{Na}_{4} \mathrm{Ir}_{3} \mathrm{O}_{8}$ : A hyper-kagome lattice antiferromagnet," Phys. Rev. B 78, 094403 (2008).

[16] G. Jackeli and G. Khaliullin, "Mott Insulators in the Strong Spin-Orbit Coupling Limit: From Heisenberg to a Quantum Compass and Kitaev Models," Phys. Rev. Lett. 102, 017205 (2009).

[17] Gang Chen, Rodrigo Pereira, and Leon Balents, "Exotic Phases Induced by Strong Spin-Orbit Coupling in Ordered Double Perovskites," Phys. Rev. B 82, 174440 (2010).

[18] W. Witczak-Krempa, G. Chen, Y. B. Kim, and L. Balents, "Correlated Quantum Phenomena in the Strong Spin-Orbit Regime," Annual Review of Condensed Matter Physics 5, $57-82$ (2014).

[19] Giniyat Khaliullin, "Orbital Order and Fluctuations in Mott Insulators," Prog. Theor. Phys. Supplement (2005), 10.1143/PTPS.160.155.

[20] Gang Chen and Leon Balents, "Spin-orbit coupling in $d^{2}$ 
ordered double perovskites," Phys. Rev. B 84, 094420 (2011).

[21] Michael Hermele, Matthew P. A. Fisher, and Leon Balents, "Pyrochlore photons: The $\mathrm{U}(1)$ spin liquid in a $\mathrm{S}=1 / 2$ three-dimensional frustrated magnet," Phys. Rev. B 69, 064404 (2004).

[22] David A. Huse, Werner Krauth, R. Moessner, and S. L. Sondhi, "Coulomb and Liquid Dimer Models in Three Dimensions," Phys. Rev. Lett. 91, 167004 (2003).

[23] Lucile Savary and Leon Balents, Phys. Rev. Lett. 108, 037202 (2012).

[24] SungBin Lee, Shigeki Onoda, and Leon Balents, "Generic quantum spin ice," Phys. Rev. B 86, 104412 (2012).

[25] Zhihao Hao, Alexandre G. R. Day, and Michel J. P. Gingras, "Bosonic many-body theory of quantum spin ice," Phys. Rev. B 90, 214430 (2014).

[26] Olga Petrova, Roderich Moessner, and S. L. Sondhi, "Hydrogenic states of monopoles in diluted quantum spin ice," Phys. Rev. B 92, 100401 (2015).

[27] Lucile Savary, Xiaoqun Wang, Hae-Young Kee, Yongbaek Kim, Yue Yu, and Gang Chen, "Quantum Spin Ice on the Breathing Pyrochlore Lattice," Phys. Rev. B 94, 075146 (2016).

[28] Nic Shannon, Olga Sikora, Frank Pollmann, Karlo Penc, and Peter Fulde, "Quantum ice: A quantum monte carlo study," Phys. Rev. Lett. 108, 067204 (2012).

[29] Gang Chen, "'magnetic monopole" condensation of the pyrochlore ice $\mathrm{u}(1)$ quantum spin liquid: Application to $\mathrm{pr}_{2} \mathrm{ir}_{2} \mathrm{O}_{7}$ and $\mathrm{yb}_{2} \mathrm{ti}_{2} \mathrm{O}_{7}$," Phys. Rev. B 94, 205107 (2016).

[30] Owen Benton, Olga Sikora, and Nic Shannon, "Seeing the light: Experimental signatures of emergent electromagnetism in a quantum spin ice," Phys. Rev. B 86, 075154 (2012).

[31] M J P Gingras and P A McClarty, "Quantum spin ice: a search for gapless quantum spin liquids in pyrochlore magnets," Reports on Progress in Physics 77, 056501 (2014).

[32] See the Supplementary Information.

[33] "Brillouin zone," Adapted from Wikipedia.

[34] Michael A. Levin and Xiao-Gang Wen, "String-net condensation: A physical mechanism for topological phases," Phys. Rev. B 71, 045110 (2005).

[35] V. K. Anand, A. K. Bera, J. Xu, T. Herrmannsdörfer, C. Ritter, and B. Lake, "Observation of long-range magnetic ordering in pyrohafnate $\mathrm{Nd}_{2} \mathrm{Hf}_{2} \mathrm{O}_{7}$ : A neutron diffraction study," Phys. Rev. B 92, 184418 (2015).

[36] E. Lhotel, S. Petit, S. Guitteny, O. Florea, M. Ciomaga Hatnean, C. Colin, E. Ressouche, M. R. Lees, and G. Balakrishnan, "Fluctuations and all-in all-out ordering in dipole-octupole $\mathrm{Nd}_{2} \mathrm{Zr}_{2} \mathrm{O}_{7}, "$ Phys. Rev. Lett. 115, 197202 (2015).

[37] A. Bertin, P. Dalmas de Réotier, B. Fåk, C. Marin, A. Yaouanc, A. Forget, D. Sheptyakov, B. Frick, C. Ritter, A. Amato, C. Baines, and P. J. C. King, " $\mathrm{Nd}_{2} \mathrm{Sn}_{2} \mathrm{O}_{7}$ : An all-in all-out pyrochlore magnet with no divergencefree field and anomalously slow paramagnetic spin dynamics," Phys. Rev. B 92, 144423 (2015).

[38] J. Xu, V. K. Anand, A. K. Bera, M. Frontzek, D. L. Abernathy, N. Casati, K. Siemensmeyer, and B. Lake, "Magnetic structure and crystal-field states of the pyrochlore antiferromagnet $\mathrm{Nd}_{2} \mathrm{Zr}_{2} \mathrm{O}_{7}$, , Phys. Rev. B 92, 224430 (2015).

[39] M. Ciomaga Hatnean, M. R. Lees, O. A. Petrenko, D. S.
Keeble, G. Balakrishnan, M. J. Gutmann, V. V. Klekovkina, and B. Z. Malkin, "Structural and magnetic investigations of single-crystalline neodymium zirconate pyrochlore $\mathrm{Nd}_{2} \mathrm{Zr}_{2} \mathrm{O}_{7}$," Phys. Rev. B 91, 174416 (2015).

[40] S. Petit, E. Lhotel, B. Canals, M. Ciomaga Hatnean, J. Ollivier, H. Mutka, E. Ressouche, A.R. Wildes, M.R. Lees, and G. Balakrishnan, "Observation of magnetic fragmentation in spin ice," Nature Physics advance online publication (2016), 10.1038/nphys3710.

[41] J. Lago, I. Živković, B. Z. Malkin, J. Rodriguez Fernandez, P. Ghigna, P. Dalmas de Réotier, A. Yaouanc, and T. Rojo, "CdEr $2 \mathrm{Se}_{4}$ : A new erbium spin ice system in a spinel structure," Phys. Rev. Lett. 104, 247203 (2010).

[42] S. Pokrzywnicki, "Analysis of the magnetic susceptibility of $\mathrm{CdYb}_{2} \mathrm{~S}_{4}$ spinel by means df the crystal field method," phys. stat. sol. (b) 71, K111 (1975).

[43] S. H. Curnoe, "Structural distortion and the spin liquid state in $\mathrm{Tb}_{2} \mathrm{Ti}_{2} \mathrm{O}_{7}$," Phys. Rev. B 78, 094418 (2008).

[44] Shigeki Onoda and Yoichi Tanaka, "Quantum fluctuations in the effective pseudospin- $\frac{1}{2}$ model for magnetic pyrochlore oxides," Phys. Rev. B 83, 094411 (2011). 


\section{Supplementary Information for "Symmetry Enriched U(1) Topological Orders for Dipole-Octupole Doublets on a Pyrochlore Lattice"}

\section{Local coordinates and the generic model}

The local coordinate system at each sublattice of the pyrochlore lattice is defined in Tab. II.

\begin{tabular}{ccccc}
\hline \hline$\mu$ & 0 & 1 & 2 & 3 \\
$\hat{x}_{\mu}$ & $\frac{1}{\sqrt{2}}[\overline{1} 10]$ & $\frac{1}{\sqrt{2}}[\overline{1} \overline{1} 0]$ & $\frac{1}{\sqrt{2}}[110]$ & $\frac{1}{\sqrt{2}}[1 \overline{1} 0]$ \\
$\hat{y}_{\mu}$ & $\frac{1}{\sqrt{6}}[\overline{1} \overline{1} 2]$ & $\frac{1}{\sqrt{6}}[\overline{1} 1 \overline{2}]$ & $\frac{1}{\sqrt{6}}[1 \overline{1} \overline{2}]$ & $\frac{1}{\sqrt{6}}[112]$ \\
$\hat{z}_{\mu}$ & $\frac{1}{\sqrt{3}}[111]$ & $\frac{1}{\sqrt{3}}[1 \overline{1} \overline{1}]$ & $\frac{1}{\sqrt{3}}[\overline{1} 1 \overline{1}]$ & $\frac{1}{\sqrt{3}}[\overline{1} \overline{1} 1]$ \\
\hline \hline
\end{tabular}

TABLE II. The local coordinate systems for the four sublattices of the pyrochlore lattice.

The dipole moment $\tau^{z}$ is defined in the local $\hat{z}$ direction, while the other two components $\tau^{x}$ and $\tau^{y}$ are defined in the pseudospin space. The magnetization of the system is thus given by

$$
\mathbf{m}=g \mu_{\mathrm{B}} \sum_{i} \tau_{i}^{z} \hat{z}_{i}
$$

where $g$ is the Landé factor and $\mu_{\mathrm{B}}$ is Bohr magneton.

To transform $H_{\mathrm{DO}}\left(H_{\mathrm{DO}}=\sum_{\langle i j\rangle}\left[J_{x} \tau_{i}^{x} \tau_{j}^{x}+J_{y} \tau_{i}^{y} \tau_{j}^{y}+\right.\right.$ $\left.\left.J_{z} \tau_{i}^{z} \tau_{j}^{z}+J_{x z}\left(\tau_{i}^{x} \tau_{j}^{z}+\tau_{i}^{z} \tau_{j}^{x}\right)\right]\right)$ to $H_{\mathrm{XYZ}}$, we perform a rotation in the pseudospin space around the local- $y$ axis,

$$
\begin{aligned}
& \tau^{x}=\cos \theta \tilde{\tau}^{x}+\sin \theta \tilde{\tau}^{z}, \\
& \tau^{y}=\tilde{\tau}^{y}, \\
& \tau^{z}=-\sin \theta \tilde{\tau}^{x}+\cos \theta \tilde{\tau}^{z},
\end{aligned}
$$

where $\tan 2 \theta=2 J_{x z} /\left(J_{z}-J_{x}\right)$. Correspondingly,

$$
\begin{aligned}
& \tilde{J}_{y}=J_{y}, \\
& \tilde{J}_{x}=\frac{1}{2}\left(J_{x}+J_{z}-\sqrt{4 J_{x z}^{2}+\left(J_{x}-J_{z}\right)^{2}}\right), \\
& \tilde{J}_{z}=\frac{1}{2}\left(J_{x}+J_{z}+\sqrt{4 J_{x z}^{2}+\left(J_{x}-J_{z}\right)^{2}}\right) .
\end{aligned}
$$

\section{Curie-Weiss temperatures}

Since the magnetization $\mathbf{m}$ is only related to the dipole moment $\tau^{z}$, the Curie-Weiss temperature only detects the interaction between $\tau^{z}$. From the original model $H_{\mathrm{DO}}$, we carry out the high temperature series expansion and find that

$$
\Theta_{\mathrm{CW}}=+\frac{J_{z}}{2}
$$

$\Theta_{\mathrm{CW}}$ does not depend the orientation of the external probing field.

\section{Perturbation theory}

Here we discuss the perturbation theory of the simplified model $H_{\text {sim }}$ with

$$
\begin{aligned}
H_{\text {sim }} & =\sum_{\langle i j\rangle} J_{y} \tau_{i}^{y} \tau_{j}^{y}-J_{ \pm}\left(\tau_{i}^{+} \tau_{j}^{-}+h . c .\right) \\
& -\sum_{i} h\left(\hat{n} \cdot \hat{z}_{i}\right) \tau_{i}^{z} .
\end{aligned}
$$

In the perturbative limit where $h \ll J_{y}$ and $J_{ \pm} \ll J_{y}$, we carry out the degenerate perturbation theory to obtain the ring exchange interaction within the ice manifold.

Without the external magnetic field, it is well-known that a third order degenerate perturbation is needed to generate the ring exchange (see Fig. 4a). Without the $J_{ \pm}$, we need a sixth order degenerate perturbation of the external magnetic field to create quantum tunneling within the octupolar ice manifold (see Fig. 4b). When both the external field and the $J_{ \pm}$terms are present, the degenerate perturbation would always involve both $J_{ \pm}$ and $h$ to generate the ring exchange. Therefore, in the ring exchange model,

$$
H_{\text {ring }}=J_{\text {ring }} \sum_{\square}\left[\tau_{i}^{+} \tau_{j}^{-} \tau_{k}^{+} \tau_{l}^{-} \tau_{m}^{+} \tau_{n}^{-}+\text {h.c. }\right] \text {, }
$$

the coupling $J_{\text {ring }}$ has the following expression,

$$
J_{\text {ring }}=\sum_{n_{1}, n_{2}} C_{n_{1}, n_{2}} h^{n_{1}}\left(-J_{ \pm}\right)^{n_{2}},
$$

where $C_{n_{1}, n_{2}}$ is a numerical coefficent in the perturbation series and $n_{1}$ is always even. The latter is because applying the Zeeman term one time only flips $\tau^{y}$ once. To get back to the ice manifold, we must always apply the Zeeman term even number of times. If the total perturbation order $n_{1}+n_{2}$ is even (odd), $C_{n_{1}, n_{2}}$ must be negative (positive). For a positive $C_{n_{1}, n_{2}}$, if $J_{ \pm}>0$, then every term in $J_{\text {ring }}$ gives a negative contribution and $J_{\text {ring }}<0$. Precisely for the same reason, the simplified model $H_{\text {sim }}$ does not have a sign problem for quantum Monte Carlo for $J_{ \pm}>0$ and for either sign of $h$.

Since the pseudospin operators $\tau_{i}^{ \pm}$in $H_{\text {ring }}$ are restricted to the spin ice manifold, we then can reexpress $\tau^{ \pm}$as

$$
\tau_{i}^{ \pm} \simeq e^{ \pm i A_{\mathbf{r r}}}
$$

where $\mathbf{r}$ and $\mathbf{r}^{\prime}$ are the centers of the two neighboring tetrahedra of the pyrochlore lattice site $i, \mathbf{r} \in$ I sublattice and $\mathbf{r}^{\prime} \in$ II sublattice, and the $2 \pi$ periodic phase variable 


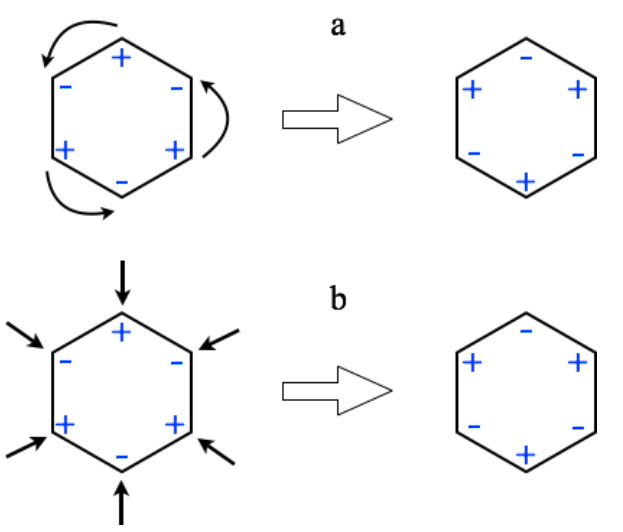

FIG. 4. The degenerate perturbation process on the elementary hexagon of the pyrochlore lattice. Here "+" and "-" represent the orientation of the $\tau^{y}$ direction. (a) The curved arrows represents applying $J_{ \pm}\left(\tau_{i}^{+} \tau_{j}^{-}+h . c\right.$.) on the bond. (b) The straight arrows represents applying $h \tau_{i}^{z}$ on the site.

$A_{\mathbf{r r}^{\prime}}$ satisfies $A_{\mathbf{r r}^{\prime}}=-A_{\mathbf{r}^{\prime} \mathbf{r}}$. After this transformation, the ring exchange becomes

$$
H_{\text {ring }} \simeq 2 J_{\text {ring }} \sum_{\bigcirc_{d}} \cos (\operatorname{curl} A),
$$

where curl $A$ is the lattice curl on the elementary hexagon $\left(\bigcirc_{d}\right)$ of the diamond lattice formed by the centers of the pyrochlore tetrahedra. Since $J_{\text {ring }}<0$, the ground state favors a zero flux with $\operatorname{curl} A=0$ on each hexagon of the diamond lattice.

\section{Gauge mean-field theory}

To implement the gauge mean-field theory [10, 23-25], we decouple the spinon-gauge coupling in the reformulated lattice gauge Hamiltonian of the main text into the spinon sector and the gauge sector. The decoupling procedure is given as follows,

$$
\begin{aligned}
\Phi_{\mathbf{r}+\eta_{\mathbf{r}} e_{\mu}}^{\dagger} & \Phi_{\mathbf{r}+\eta_{\mathbf{r}} e_{\nu}} s_{\mathbf{r}, \mathbf{r}+\eta_{\mathbf{r}} e_{\mu}}^{-\eta_{\mathbf{r}}} s_{\mathbf{r}, \mathbf{r}+\eta_{\mathbf{r}} e_{\nu}}^{+\eta_{\mathbf{r}}} \rightarrow\left\langle s_{\mathbf{r}, \mathbf{r}+\eta_{\mathbf{r}} e_{\mu}}^{-\eta_{\mathbf{r}}}\right\rangle\left\langle s_{\mathbf{r}, \mathbf{r}+\eta_{\mathbf{r}} e_{\nu}}^{+\eta_{\mathbf{r}}}\right\rangle \\
& \times\left[\Phi_{\mathbf{r}+\eta_{\mathbf{r}} e_{\mu}}^{\dagger} \Phi_{\mathbf{r}+\eta_{\mathbf{r}} e_{\nu}}-\left\langle\Phi_{\mathbf{r}+\eta_{\mathbf{r}} e_{\mu}}^{\dagger} \Phi_{\mathbf{r}+\eta_{\mathbf{r}} e_{\nu}}\right\rangle\right] \\
& +\left[\left\langle s_{\mathbf{r}, \mathbf{r}+\eta_{\mathbf{r}} e_{\mu}}^{-\eta_{r}}\right\rangle s_{\mathbf{r}, \mathbf{r}+\eta_{\mathbf{r}} e_{\nu}}^{+}+s_{\mathbf{r}, \mathbf{r}+\eta_{\mathbf{r}} e_{\mu}}^{-\eta_{\mathbf{r}}}\left\langle s_{\mathbf{r}, \mathbf{r}+\eta_{\mathbf{r}} e_{\nu}}^{+\eta_{\mathbf{r}}}\right\rangle\right. \\
& \left.-\left\langle s_{\mathbf{r}, \mathbf{r}+\eta_{\mathbf{r}} e_{\mu}}^{-\eta_{\mathbf{r}}}\right\rangle\left\langle s_{\mathbf{r}, \mathbf{r}+\eta_{\mathbf{r}} e_{\nu}}^{+\eta_{\mathbf{r}}}\right\rangle\right]\left\langle\Phi_{\mathbf{r}+\eta_{\mathbf{r}} e_{\mu}}^{\dagger} \Phi_{\mathbf{r}+\eta_{\mathbf{r}} e_{\nu}}\right\rangle, \quad(25) \\
\Phi_{\mathbf{r}}^{\dagger} \Phi_{\mathbf{r}^{\prime}} s_{\mathbf{r r}^{\prime}}^{+} & \rightarrow\left[\Phi_{\mathbf{r}}^{\dagger} \Phi_{\mathbf{r}^{\prime}}-\left\langle\Phi_{\mathbf{r}}^{\dagger} \Phi_{\mathbf{r}^{\prime}}\right\rangle\right]\left\langle s_{\mathbf{r r}^{\prime}}^{+}\right\rangle+\left\langle\Phi_{\mathbf{r}}^{\dagger} \Phi_{\mathbf{r}^{\prime}}\right\rangle s_{\mathbf{r r}^{\prime}}^{+} .
\end{aligned}
$$

With the above decoupling, the gauge sector is trivially solved for the zero gauge flux sector, and we have $s \equiv$ $\left\langle s_{\mathbf{r r}^{\prime}}^{ \pm}\right\rangle=1 / 2$ on every link of the diamond lattice. For the spinon sector, the spinon mean-field Hamiltonian is now reduced to

$$
\begin{aligned}
H_{\text {spinon }}= & \frac{J_{y}}{2} \sum_{\mathbf{r}} Q_{\mathbf{r}}^{2}-J_{ \pm} s^{2} \sum_{\mathbf{r}} \sum_{\mu \neq \nu} \Phi_{\mathbf{r}+\eta_{\mathbf{r}} \mathbf{e}_{\mu}}^{\dagger} \Phi_{\mathbf{r}+\eta_{\mathbf{r}} \mathbf{e}_{\nu}} \\
& -\frac{h s}{2} \sum_{\left\langle\mathbf{r} \mathbf{r}^{\prime}\right\rangle}\left(\hat{n} \cdot \hat{z}_{i}\right)\left(\Phi_{\mathbf{r}}^{\dagger} \Phi_{\mathbf{r}^{\prime}}+\text { h.c. }\right) .
\end{aligned}
$$

It is convenient to introduce a rotor variable $\phi_{\mathbf{r}}$ such that $\left[\phi_{\mathbf{r}}, Q_{\mathbf{r}^{\prime}}\right]=i \delta_{\mathbf{r r}^{\prime}}$. Then we have $\Phi_{\mathbf{r}}=e^{-i \phi_{\mathbf{r}}}$ and $\left|\Phi_{\mathbf{r}}\right|=1$. After such a transformation, the electric charge density $Q_{\mathbf{r}}$ can take any integer value. This approximation is legitimate since the weight with large $Q_{\mathbf{r}}$ is suppressed by the antiferromagnetic $J_{y}$. We further carry out the standard procedure and implement a coherence state path integral for the phase rotor variable. We integrate out $Q_{\mathbf{r}}$ and obtain the partition function

$$
\mathcal{Z}=\int \mathcal{D} \Phi^{\dagger} \mathcal{D} \Phi \mathcal{D} \lambda e^{-\mathcal{S}-\sum_{\mathbf{r}} \int d \tau \lambda_{\mathbf{r}}\left(\left|\Phi_{\mathbf{r}}\right|^{2}-1\right)},
$$

where the effective action $\mathcal{S}$ is given by

$$
\begin{aligned}
\mathcal{S}= & \int d \tau \sum_{\mathbf{r}} \frac{\left|\partial_{\tau} \Phi_{\mathbf{r}}\right|^{2}}{2 J_{y}}-J_{ \pm} s^{2} \sum_{\mathbf{r}} \sum_{\mu \neq \nu} \Phi_{\mathbf{r}+\eta_{\mathbf{r}} \mathbf{e}_{\mu}} \Phi_{\mathbf{r}+\eta_{\mathbf{r}} \mathbf{e}_{\nu}} \\
& -\frac{h s}{2} \sum_{\left\langle\mathbf{r r}^{\prime}\right\rangle}\left(\hat{n} \cdot \hat{z}_{i}\right)\left(\Phi_{\mathbf{r}}^{\dagger} \Phi_{\mathbf{r}^{\prime}}+\text { h.c. }\right),
\end{aligned}
$$

and $\lambda_{\mathbf{r}}$ is introduced to impose the unimodular constraint $\left|\Phi_{\mathbf{r}}\right|=1$. With a uniform saddle point approximation by setting $\lambda_{\mathbf{r}}=\lambda$, we obtain two spinon dispersions,

$$
\begin{aligned}
& \omega_{1}(\mathbf{k})=\left[2 J_{y}\left(\lambda-J_{ \pm} L_{1}(\mathbf{k})+h\left|L_{2}(\mathbf{k})\right|\right)\right]^{1 / 2}, \\
& \omega_{2}(\mathbf{k})=\left[2 J_{y}\left(\lambda-J_{ \pm} L_{1}(\mathbf{k})-h\left|L_{2}(\mathbf{k})\right|\right)\right]^{1 / 2},
\end{aligned}
$$

where

$$
\begin{aligned}
& L_{1}(\mathbf{k})=s^{2} \sum_{i=1}^{12} \cos \left(\mathbf{k} \cdot \mathbf{a}_{i}\right), \\
& L_{2}(\mathbf{k})=\frac{s}{2} \sum_{\mu=0}^{3}\left(\hat{z}_{\mu} \cdot \hat{n}\right) e^{i \mathbf{k} \cdot \mathbf{e}_{\mu}} .
\end{aligned}
$$

Here $\left\{\mathbf{a}_{i}\right\}$ are twelve second-neighbor vectors of the diamond lattice. The parameter $\lambda$ is solved by the selfconsistent equation $\left\langle\Phi_{\mathbf{r}}^{\dagger} \Phi_{\mathbf{r}}\right\rangle=1$ with

$$
\sum_{\mathbf{k}}\left[\frac{J_{y}}{\omega_{1}(\mathbf{k})}+\frac{J_{y}}{\omega_{2}(\mathbf{k})}\right]=2 .
$$

\section{Distinction between the dipolar U(1) QSLs for DO doublets and usual Kramers' doublets}

Here we explain the difference between the dipolar U(1) QSL for DO doublets and the dipolar U(1) QSL for the usual Kramers' doublets. For the usual Kramers' doublets, the generic exchange Hamiltonian is $[23,24,43,44]$

$$
\begin{aligned}
H_{\text {Kramers }} & =\sum_{\langle i j\rangle} J_{z z} S_{i}^{z} S_{j}^{z}-J_{ \pm}\left(S_{i}^{+} S_{j}^{-}+S_{i}^{-} S_{j}^{+}\right) \\
& +J_{ \pm \pm}\left[\gamma_{i j} S_{i}^{+} S_{j}^{+}+\gamma_{i j}^{*} S_{i}^{-} S_{j}^{-}\right] \\
& +J_{z \pm}\left[S_{i}^{z}\left(\zeta_{i j} S_{j}^{+}+\zeta_{i j}^{*} S_{j}^{-}\right)+(i \leftrightarrow j)\right],
\end{aligned}
$$


where $\gamma_{i j}$ is bond dependent phase factor that takes $1, e^{i 2 \pi / 3}, e^{-i 2 \pi / 3}$ on different bonds, $\zeta_{i j}=-\gamma_{i j}^{*}$, and $S_{i}^{ \pm}=S_{i}^{x} \pm i S_{i}^{y}$. Please note the difference of $S^{ \pm}$from the definitiion of $\tau^{ \pm}$in the main text. In the parameter regime with $J_{z z} \gg\left|J_{ \pm}\right|,\left|J_{ \pm \pm}\right|,\left|J_{z \pm}\right|$ and the neighboring parameter regime, the ground state of $H_{\text {Kramers }}$ is the dipolar U(1) QSL where the the Ising component $S^{z}$ behaves as the emergent electric field and the transverse components $S^{ \pm}$create spinon excitations. All the spin components of an usual Kramers' doublet are magnetic dipole moments, thus all of them couple linearly with the external magnetic field and the neutron spin. Therefore, the inelastic neutron scattering detects both the gapped spinon continuum and the gapless gauge phonon in the dipolar U(1) QSL for the usual Kramers doublets.

For the DO doublet, the generic model is given by
$H_{\mathrm{DO}}$. This model can be obtained from $H_{\text {Kramers }}$ if one simply sets $\gamma_{i j}$ and $\zeta_{i j}$ to 1 on every bond, but the ground states of $H_{\mathrm{DO}}$ cannot be obtained from $H_{\text {Kramers }}$ in this manner. As we have described in the main text, what we have done is to perform a rotation about the $y$ axis in the pseudospin space to eliminate the crossing term $J_{x z}$. The resulting model is the XYZ model.

Let us here focus on the dipolar U(1) QSL in the regime $\tilde{J}_{z} \gg\left|\tilde{J}_{x}\right|,\left|\tilde{J}_{y}\right|$. In this phase, $\tilde{\tau}^{z}$ is the emergent electric field and $\tilde{\tau}^{x}$ creates the spinon excitations. The external magnetic field and the neutron spin couple linearly to the $\tau^{z}$ component. Since $\tau^{z}$ is a combination of $\tilde{\tau}^{x}$ and $\tilde{\tau}^{z}$, the external magnetic field and the neutron spin couple with both the emergent electric field and the spinons. For this reason, the inelastic neutron scattering measurement detects both the gapless gauge photon and the gapped spinon continuum. This is clearly different from the origin for the usual Kramers' doublets. 